Vanderlei C da Silva'

Paulo 0 Scherer"I

Simone S Falcão"

Jeronimo Alencar'

Sergio P Cunha'II

Iram M Rodrigues'v

Nadja L Pinheiro"

\section{Diversidade de criadouros e tipos de imóveis freqüentados por Aedes albopictus e Aedes aegypti}

\section{Diversity of oviposition containers and buildings where Aedes albopictus and Aedes aegypti can be found}

\section{RESU MO}

OBJETIVOS: Verificar a diversidade de criadouros e tipos de imóveis freqüentados por fêmeas de Aedes albopictus e Aedes aegypti.

MÉTODOS: O estudo foi realizado nos anos de 2002 e 2003 no bairro de Campo Grande, Rio de Janeiro, RJ. Realizou-se pesquisa larvária em diferentes tipos de imóveis. As larvas encontradas foram identificadas em laboratório. A freqüência de larvas dessas duas espécies foi computada nos diversos criadouros disponíveis. Foram calculados os índices de infestação predial e de Breteau, as diferenças foram testadas pelo qui-quadrado.

RESULTADOS: Os tipos de imóveis positivos para os aedinos foram: residências (83,9\% do total); igrejas, escolas, clubes $(6,8 \%)$; terrenos baldios $(6,4 \%)$; e comércios (2,8\%). Das 9.153 larvas, 12,0\% eram de Aedes albopictus e 88,0\% de Aedes aegypti. Para aquela espécie, os recipientes onde foram mais encontradas foram ralos $(25,4 \%)$, latas, garrafas, vasilhames $(23,9 \%)$ e vasos com plantas $(16,2 \%)$. Aedes aegypti mostrou-se mais frequiente nos criadouros que Aedes albopictus $\left(\chi^{2}=145,067, \mathrm{p}<0,001\right)$. Também ocorreu diferença significante na freqüência dessas espécies em criadouros artificiais do que em naturais $\left(\chi^{2}=31,46 ; p<0,001\right)$. O índice de infestação predial e índice de Breteau para Aedes albopictus foram respectivamente em $2002(0,3 \%$; 0,28), em 2003 (0,4\%; 0,5); para Aedes aegypti, em $2002(1,0 \% ; 1,16)$ e $2003(3,5 \% ; 4,35)$.

CONCLUSÕES: Verificou-se a freqüência das fêmeas de Aedes albopictus e Ae. aegypti em variados tipos de criadouros e tipos de imóveis para postura. A oferta abundante de recipientes artificiais inservíveis nas residências, associada à capacidade de Ae. albopictus de freqüentar também os criadouros naturais, contribui sobremaneira para sua adaptação gradativa ao meio antrópico.

DESCRITO RES: Aedes aegypti. Aedes albopictus. Larva, crescimento e desenvolvimento. Distribuição espacial da população. Insetos vetores.

\section{ABSTRACT \\ ABSTRACT}

Vanderlei C. da Silva

Departamento de Entomologia - Fiocruz

Av. Brasil, 4365

21040-900 Rio de Janeiro, RJ, Brasil

E-mail: camposil@ioc.fiocruz.br
OBJECTIVE: To assess the diversity of oviposition containers and buildings where females of Aedes albopictus and Aedes aegypti can be found.

METHODS: A study was carried out in the city of Rio de Janeiro, Southern Brazil, 
between 2002 and 2003. Larvae in different types of buildings were investigated, and immature forms found were then sent to the laboratory for identification. The larval frequency for both mosquitoes was estimated in the oviposition containers available. The Breteau index and the building infestation index were calculated and differences were tested using the Chi-square test.

RESULTS: The types of buildings that were positive for Aedes albopictus and Aedes aegypti were: dwellings (83.9\%); churches, schools, clubs (6.8\%); vacant land (6.4\%); and businesses (2.8\%). Of 9,153 larvae collected, $12.0 \%$ were Aedes albopictus and $88.0 \%$ were Aedes aegypti. Aedes albopictus were mostly found in drains (25.4\%); cans, bottles, empty bottles (23.9\%); and plant vases (16.2\%). Aedes aegypti was much more frequently found than Aedes albopictus $\left(\chi^{2}=145.067 ; \mathrm{p}<0.001\right)$. Both species were significantly more frequent in artificial than in natural oviposition containers $\left(\chi^{2}=31.46 ; \mathrm{p}<0.001\right)$. The building infestation index and Breteau index for Aedes albopictus were $0.3 \%$ and $0.28 \%$ in 2002 and $0.4 \%$ and 0.5 in 2003, respectively. For Aedes aegypti, they were $1.0 \%, 1.16$ in 2002 and $3.5 \%$ and 4.35 in 2003, respectively.

CONCLUSIONS: The present study assessed the frequencies of Aedes albopictus and Aedes aegypti females in various types of oviposition containers and types of buildings. The abundant availability of artificial containers in dwellings, associated with the capacity of Ae. albopictus to be also found in natural oviposition containers, has greatly contributed for their gradual adaptation to human environment.

\section{KEYWO RDS: Aedes aegypti. Aedes albopictus. Larva, growth \& development. Spatial distribution of population. Insect vectors.}

\section{INTRODUÇÃO}

Aedes albopictus, originalmente descrito da Índia, possui uma elevada capacidade de dispersão, utilizando-se tanto dos recipientes naturais quanto dos artificiais para postura, em ambiente urbano ou silvestre. No continente americano o registro do aparecimento dessa espécie ocorreu no sul dos Estados Unidos, em 1985 (Sprenger \& Wuithranyagool, ${ }^{12}$ 1986), enquanto que no território brasileiro foi primeiramente encontrado nos Estados do Rio de Janeiro e Minas Gerais, em 1986 (Forattini, ${ }^{4}$ 1986). Desde sua introdução no País, apenas sete estados brasileiros ainda não relataram infestação pelo Ae. albopictus: Amapá, Roraima, Acre, Tocantins, da região Norte; e Piauí, Ceará e Sergipe, da região Nordeste (Santos, $\left.{ }^{10} 2003\right)$. Este díptero é eclético no que diz respeito ao exercício da hematofagia, podendo exercê-la em um número grande de animais, inclusive acentuada antropofilia (Marques \& Gomes, ${ }^{7}$ 1997).

No que concerne à dengue, uma das doenças virais mais importantes do mundo, o Ae. albopictus não é incriminado como vetor no Brasil. A distribuição e abundância desse mosquito não coincidem com a da doença, muito embora experimentalmente, demonstre susceptibilidade e capacidade de transmitir os quatro sorotipos do vírus (Consoli \& Lourenço-de-Oliveira, ${ }^{2}$ 1994). Por freqüentar igualmente os meios urbano e silvestre, esta espécie pode ser ca- paz de veicular agentes patogênicos entre esses ambientes.

O presente trabalho teve como objetivo registrar o comportamento de Ae. albopictus e Ae. aegypti frente à diversidade de criadouros e tipos de imóveis, comparando-se as duas espécies.

\section{MÉTO DOS}

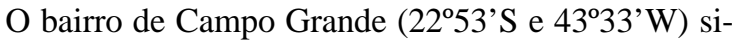
tua-se na zona oeste e dista cerca de $50 \mathrm{~km}$ do centro do município do Rio de Janeiro. Possui uma área territorial de $171,67 \mathrm{~km}^{2}$ e, segundo o Instituto Brasileiro de Geografia e Estatística, possui 297.494 habitantes (Figura).

A Secretaria Municipal de Saúde, por meio do Programa Municipal de Controle da Dengue, realizou nos anos de 2002 e 2003 coletas sistemáticas de formas imaturas de Culicidae nas residências e em outros tipos de imóveis da área de estudo. As formas imaturas eram acondicionadas em tubo de hemólise contendo álcool a 70\% e enviadas ao laboratório de entomologia para identificação. Apenas as amostras que continham larvas e pupas de Aedes aegypti e/ou Aedes albopictus eram consideradas positivas. Tipos de criadouros, imóveis, quantidades de quarteirões, e outras informações, eram também anotados para posteriormente serem analisados. 
As larvas coletadas eram registradas em boletins próprios, que alimentavam um banco de dados de uso nacional, o sistema de informação de febre amarela e dengue da Secretaria de Vigilância em Saúde. Com estas informações obtinham-se o índice de infestação predial, básico para a verificação do grau de infestação de uma localidade qualquer, e índice de Breteau dos bairros. Para obter o valor do índice de infestação predial, divide-se o número de imóveis positivos para uma espécie pelo número de imóveis inspecionados, e o resultado é multiplicado por 100 (Funasa, * 1998). O índice de Breteau é utilizado para estimar a densidade larvária do $A e$. aegypti ou Ae. albopictus, mas não considera a produtividade de criadouros (Gomes, ${ }^{6}$ 1998). O valor desse índice é obtido dividindo-se o número de recipientes positivo pelo número de imóveis inspecionado, multiplicando-se o resultado por 100 .

O período de estudo foi dividido em ciclos operacionais que representavam o intervalo de tempo entre uma visita e outra do agente

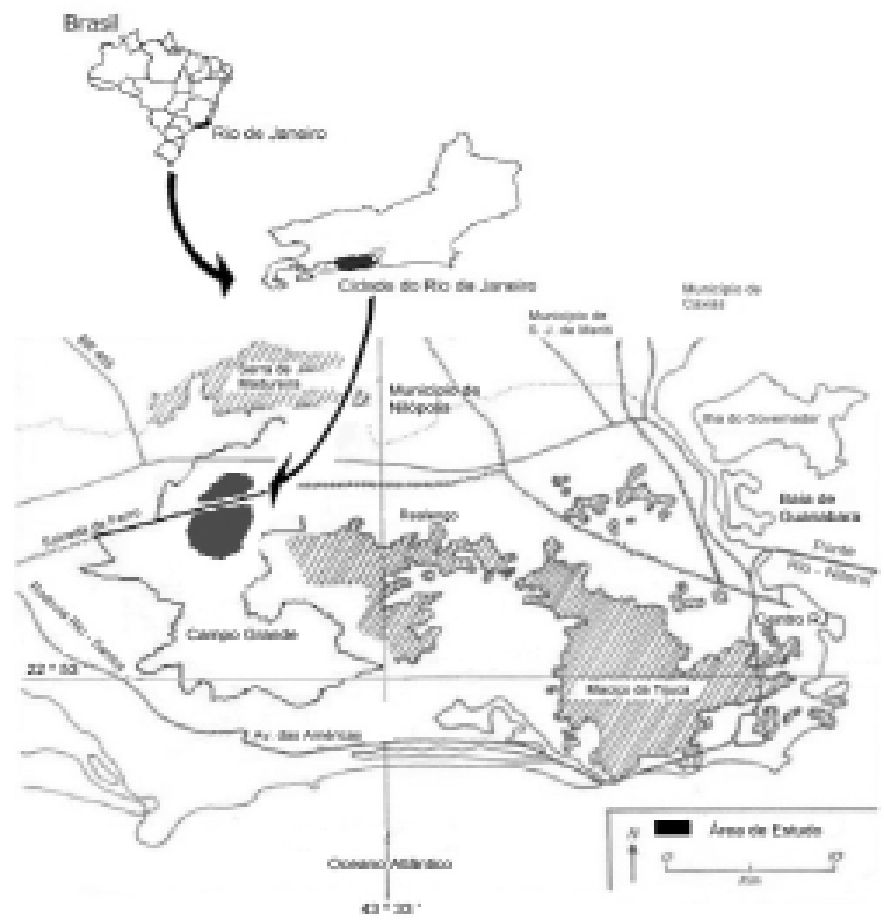

Figura - Localização geográfica da área de estudo. de saúde no mesmo imóvel. Os dados de 2002 processados foram obtidos no segundo e terceiro ciclos, correspondendo aos períodos de 5/5 a 6/7 e 7/7 a 1/9, respectivamente. No ano de 2003, os dados representam aqueles obtidos no quarto e quinto ciclos operacionais, de $3 / 8$ a $18 / 10$ e 19/10/2003 a 3/1/2004, respectivamente. As coletas foram delineadas de modo a apresentarem significância e garantir assim, uma representação na pesquisa larvária em todos os quarteirões e imóveis dessa localidade. Segundo a Fundação Nacional de Saúde (Funasa,* 1998), "quarteirão" deve ser entendido como sendo um espaço determinado por um agrupamento de imóveis, limitados por ruas, avenidas, rios, linha férrea e outros desde que seja possível circundá-lo. Os depósitos que continham água no seu interior, por sua vez, eram inspecionados, cuidadosamente, tratados com larvicida e/ou eliminado, conforme orientação contida no manual de normas técnicas da Funasa* (1998). Esses recipientes são classificados em: caixa d'água (qualquer depósito de água colocado em um nível elevado, permitindo assim a distribuição desta pela gravidade); tanque (reservatório com água colocado ao nível do solo); depósito de barro (construído com esse material.); depósito de madeira (barril, tonel e tinas); pneus; recipientes naturais (coleções de água encontradas em cavidades de árvores e imbricações de folhas); cacimbas, poços e cisternas (escavações feitas no solo) e outros (ralos).

Para análise dos dados utilizou-se o teste do qui-quadrado $\left(\chi^{2}\right)$, para comparar a frequiência da presença de larvas de Ae. aegypti e Ae. albopictus nos criadouros.

\section{RESU LTADOS}

Foram inspecionados 269.317 imóveis, dos quais 42,6\% foram trabalhados em 2002 e 57,4\% em 2003. No que diz respeito ao número de quarteirões, foram investigados 7.816 , sendo $48,2 \%$ em 2002 e 51,8\% em 2003.

Quanto aos tipos de imóveis, as residências aparecem com grande destaque na "preferência" das fêmeas de Ae. aegypti e Ae. albopictus na hora da postura. No ano de 2002 essas moradias representaram 79,1\% dos imóveis infestados, e em 2003 esse índice subiu para $85,1 \%$. Terrenos baldios apresentavam índice de 10,1\% em 2002 contra 5,4\% em 2003. As escolas, clubes e igrejas, encontram-se representados com 6,0\% e 7,0\% no período seguinte. Finalmente, os índices dos comércios foram de 4,0\% no primeiro ano e em 2003, para 2,5\% (Tabela 1).

Das 9.153 larvas coletadas, $88,0 \%$ eram Ae. aegypti $\mathrm{e}$ $12,0 \%$ de Ae. albopictus, distribuídos ao longo de 2.429 quarteirões positivos para pelo menos uma das espécies. O Ae. aegypti foi novamente a espécie predominante nos dois anos de coleta, comparecendo 
Tabela 1 - Tipos de imóveis infestados com Ae. aegypti e/ou Ae. albopictus. Rio de Janeiro, RJ, 2002-2003.

\begin{tabular}{|c|c|c|c|c|c|}
\hline \multirow[t]{2}{*}{ Tipo de imóvel } & \multicolumn{2}{|c|}{2002} & \multicolumn{2}{|c|}{2003} & \multirow[t]{2}{*}{ Total } \\
\hline & $\mathrm{N}$ & $\%$ & $\mathrm{~N}$ & $\%$ & \\
\hline \multirow[t]{2}{*}{$\begin{array}{l}\text { Residencial } \\
\text { Comercial } \\
\text { Terreno baldio } \\
\text { Outros }\end{array}$} & $\begin{array}{l}1.176 \\
59 \\
162 \\
89\end{array}$ & $\begin{array}{c}79,1 \\
4,0 \\
10,9 \\
6,0\end{array}$ & $\begin{array}{l}5.219 \\
155 \\
328 \\
432\end{array}$ & $\begin{array}{l}85,1 \\
2,5 \\
5,3 \\
7,0\end{array}$ & $\begin{array}{l}6.395 \\
214 \\
490 \\
521\end{array}$ \\
\hline & 1.486 & 100,0 & 6.134 & 100,0 & 7.620 \\
\hline
\end{tabular}

Tabela 2 - Número de quarteirões positivos por pelo menos uma das espécies, segundo ano de coleta. Rio de Janeiro, RJ, 20022003.

\begin{tabular}{cccccccc}
\hline Ano & Ae. aegypti & $\%$ & Ae. albopictus & $\%$ & Ambas & $\%$ & Total \\
\hline 2002 & 531 & 26,9 & 29 & 35,4 & 94 & 25,3 & 654 \\
2003 & 1.445 & 73,1 & 53 & 64,6 & 277 & 74,7 & 1.775 \\
\hline & 1.976 & 100,0 & 82 & 100,0 & 371 & 100,0 & 2.429 \\
\hline
\end{tabular}

com $81,3 \%$ contra $3,4 \%$ do Ae. albopictus. Ambas as espécies estiveram presentes em $15,3 \%$ desses quarteirões (Tabela 2).

Foram inspecionados mais de um milhão de depósitos, dos quais $811.472(77,4 \%)$ foram tratados com larvicida e/ou eliminado, de forma a não oferecer meios de proliferação do mosquito.

No que concerne ao encontro de ambas às espécies nos recipientes computados, Ae. aegypti mostrou-se bem mais freqüente que Ae. albopictus $\left(\chi^{2}=145,067\right.$, $\mathrm{p}<0,001)$. Também ocorreu diferença significante $\left(\chi^{2}=31,46 ; p<0,001\right)$ na freqüência das duas espécies em relação ao tipo de criadouro, sendo maior nos artificiais que nos naturais (incluindo as bromélias). $\mathrm{O}$ Aedes albopictus mostrou-se, como era de se esperar, bastante eclético na freqüência aos criadouros para oviposição, principalmente aqueles localizados no peridomicílio. Os criadouros mais freqüentados para esta espécie foram os ralos $(25,4 \%)$, seguido pelas latas, garrafas e vasilhames de plástico $(23,9 \%)$, e os recipientes naturais, $6,0 \%$. As peças de carros e materiais de construções, incluídos no registro devido a alta concentração desses objetos na área de estudo, ficou com 4,3\%, as caixas d'água com $4,2 \%$ e por último as bromélias, que apresentaram frequiência de 3,1\% (Tabela 3).
As fêmeas de Ae. aegypti parecem ter a mesma "preferência" observada para Ae. albopictus no que diz respeito aos criadouros artificiais para oviposição. A freqüência de positividade para Ae. aegypti foi de 26,3\% para os ralos, e 25,3\% para vasos com planta. As garrafas, latas e plásticos representaram 16,2\% dos positivos, e os tambores, tinas e depósitos de barro, muito utilizados para o armazenamento de água, foram responsáveis por $10,6 \%$ do total de focos. Outros depósitos importantes apresentaram percentuais relativamente pequenos: caixas d'água $(7,0 \%)$, pneus $(4,4 \%)$ e peças para construção $(4,4 \%)$. Os depósitos naturais representaram $3,5 \%$ e as bromélias, $1,5 \%$.

O índice de infestação predial (IIP) e índice de Breteau (IB) para Ae. albopictus, foram respectivamente de $0,3 \%$ e 0,28 (2002) e em 2003, estes mesmos índices foram de $0,4 \%$ e 0,5 . Para Ae. aegypti esses parâmetros foram IIP $=1,0 \%$ e IB $=1,16$ em 2002 e IIP $=3,5 \%$ e $\mathrm{IB}=4,4$ em 2003 (Tabela 4).

\section{DISCUSSÃO}

Para todos os criadouros computados o Ae. albopictus apresentou leve tendência a realizar suas posturas nos depósitos localizados no peridomicílio. Tal aspecto de comportamento deve-se, provavelmente, à caracte-

Tabela 3 - Larvas de Ae. aegypti e Ae. albopictus por tipo de recipiente. Rio de Janeiro, RJ, 2002-2003.

\begin{tabular}{|c|c|c|c|c|c|}
\hline \multirow[t]{2}{*}{ Tipo de depósitos } & \multicolumn{2}{|c|}{ Ae. aegypti } & \multicolumn{2}{|c|}{ Ae. albopictus } & \multirow{2}{*}{$\begin{array}{c}\text { Total } \\
\mathrm{N}\end{array}$} \\
\hline & $\mathrm{N}$ & $\%$ & $\mathrm{~N}$ & $\%$ & \\
\hline \multirow[t]{2}{*}{$\begin{array}{l}\text { Pneu } \\
\text { Tambor, tanque, barril, depósito barro } \\
\text { Vaso com planta } \\
\text { Peça de carro, material de construção } \\
\text { Garrafa, lata, plástico } \\
\text { Poço, cisterna, cacimba } \\
\text { Caixa d'água } \\
\text { Recipiente natural } \\
\text { Ralos } \\
\text { Armadilhas } \\
\text { Bromélias }\end{array}$} & $\begin{array}{c}353 \\
850 \\
2.034 \\
350 \\
1.306 \\
71 \\
567 \\
283 \\
2.120 \\
1 \\
119\end{array}$ & $\begin{array}{c}4,4 \\
10,6 \\
25,3 \\
4,3 \\
16,2 \\
0,9 \\
7,0 \\
3,5 \\
26,3 \\
0,0 \\
1,5\end{array}$ & $\begin{array}{c}90 \\
87 \\
178 \\
47 \\
263 \\
9 \\
46 \\
66 \\
279 \\
0 \\
34\end{array}$ & $\begin{array}{c}8,2 \\
7,9 \\
16,2 \\
4,3 \\
23,9 \\
0,8 \\
4,2 \\
6,0 \\
25,4 \\
0,0 \\
3,1\end{array}$ & $\begin{array}{c}443 \\
937 \\
2.212 \\
397 \\
1.569 \\
80 \\
613 \\
349 \\
2.399 \\
1 \\
153\end{array}$ \\
\hline & 8.054 & 100,0 & 1.099 & 100,0 & 9.153 \\
\hline
\end{tabular}


Tabela 4 - Total de imóveis inspecionados, número de recipientes e imóveis positivos e índices de infestação para Ae. aegypti e Ae. albopictus. Rio de Janeiro, RJ, 2002-2003.

\begin{tabular}{|c|c|c|c|c|c|}
\hline Ano/espécie In & Imóveis inspecionados & Recipiente + & Imóveis + & $\operatorname{IP}(\%)$ & $\mathrm{IB}$ \\
\hline $\begin{array}{l}2002 \\
\text { Ae. aegypti } \\
\text { Ae. albopictus } \\
\text { Total }\end{array}$ & $\begin{array}{c}32.476 \\
82.230 \\
114.706\end{array}$ & $\begin{array}{c}1.326 \\
326\end{array}$ & $\begin{array}{c}1.192 \\
294\end{array}$ & $\begin{array}{l}1,0 \\
0,3\end{array}$ & $\begin{array}{l}1,16 \\
0,26\end{array}$ \\
\hline $\begin{array}{l}2003 \\
\text { Ae. aegypti } \\
\text { Ae. albopictus } \\
\text { Total }\end{array}$ & $\begin{array}{c}77.545 \\
77.066 \\
154.611\end{array}$ & $\begin{array}{c}6.728 \\
773\end{array}$ & $\begin{array}{c}5.451 \\
683\end{array}$ & $\begin{array}{l}3,5 \\
0,4\end{array}$ & $\begin{array}{l}4,35 \\
0,50\end{array}$ \\
\hline
\end{tabular}

IP: Índice predial

IB: Índice de Breteau

rística semidoméstica peculiar desta espécie (Chiaravalloti-Neto et al, ${ }^{1}$ 1996). Na região de São José do Rio Preto, Chiaravalloti-Neto, também mencionou um maior grau de associação entre o Ae. albopictus com os criadouros naturais e os materiais descartáveis pelo homem (latas, potes, frascos, copos, e outros tipos de recipientes removíveis). A farta oferta destes últimos criadouros, quase sempre provenientes de um descarte inadequado, aliado à característica desta espécie de distribuir seus ovos entre vários depósitos, faz com que ela se disperse facilmente.

Separados dos recipientes naturais pelo programa de controle da dengue, as Bromeliaceae possuem propriedade de acumular água em seu reservatório (tanque), e conseqüentemente possibilitam albergar formas imaturas de culicideos. Marques \& Forattini ${ }^{8}$ (2005) verificaram que a capacidade volumétrica das bromélias em ambiente urbano é maior que as de ambientes periurbano e silvestres e também mais positivas. As bromélias se destacam ainda pelo fato de representarem um importante elemento na composição de paisagismos no meio urbano, trazendo, assim, preocupação a mais para o programa de controle da dengue (Cunha, ${ }^{3}$ 2002). Isso porque a domesticação dessas plantas acrescenta mais uma opção de criadouro viável tanto para o Ae. aegypti quanto para o Ae. albopictus. Atente-se ao fato de que esta última espécie mostra-se mais afeita a esses tipos de criadouro.

Aedes aegypti e Aedes albopictus apresentam comportamento semelhante no que se refere à frequiência de suas formas imaturas nos diversos criadouros artificiais. Com isso, as três categorias com maior positividade (ralos, vasos com plantas e latas, garrafas e plásticos) representaram quase $70 \%$ do total contra $32,5 \%$ do total de larvas coletadas. Este alto índice provavelmente se deve à grande concentração de utensílios e demais objetos utilizados pelas donas de casa no seu dia-a-dia, e à forma inadequada na hora do descarte desse material, o que faz com que os programas de controle dirijam suas ações para esses depósitos.
Os índice de infestação predial e de Breteau para as duas espécies mostraram-se semelhantes. O IIP de Ae. aegypti teve queda do segundo para o terceiro ciclo operacional em 2002, porém voltou a subir em 2003 nos ciclos seguintes até chegar a 4,0\% no final da pesquisa. Esse índice é considerado como situação de iminente perigo à saúde pública. Também ocorreu aumento nesse índice para Ae. albopictus, embora o percentual tenha permanecido abaixo de um, assim como o IB. O índice de Breteau de Ae. aegypti aumentou, chegando ao final do período com 5,09 recipientes positivos para cada 100 domicílios pesquisados (Tabela 3).

Em relação ao significado epidemiológico, Forattini ${ }^{5}$ (2002) considera que o Ae. albopictus merece vigilância. Esse mosquito mostrou-se, em experimentos laboratoriais, capaz de infectar-se com cerca de 23 arbovírus (Moore \& Mitchell, ${ }^{9}$ 1997), inclusos aí os vírus da dengue e da febre amarela.

Ainda, esse mosquito pode se tornar uma ponte entre os ciclos silvestre e urbano da febre amarela e de outros arbovírus no Brasil, considerando-se sua facilidade de freqüentar igualmente os ambientes silvestres, rurais, suburbanos e urbanos (Consoli \& Lourenço-de-Oliveira, ${ }^{2}$ 1994). Entretanto, populações de Ae. albopictus do Brasil ainda não foram incriminadas como vetor de dengue ou outro arbovírus, pois a sua distribuição e abundância não coincidem com a doença (Serufo et al, ${ }^{11}$ 1993).

Se ocorrer a comprovação de que Ae. albopictus é vetor eficaz da febre amarela, representará um problema de alta gravidade, pois é ainda duvidoso que haja alguma expectativa de controlar populações, no momento em que esta espécie se estabelecer em ambiente silvestre.

As residências apresentaram uma grande concentração de recipientes descartados em seu entorno, gerando, conseqüentemente, novos focos de mosquitos transmissores da dengue. Entretanto não haverá controle ou erradicação desses vetores, se não houver uma real conscientização da população no sentido 
de educar e destinar com maior eficácia os materiais desprezados diariamente pelo homem. É preciso orientar o público, principalmente com campanhas pu- blicitárias, veiculadas nos diversos tipos de comunicação, enfocando a gravidade com que a dengue vem afetando a população brasileira.

\section{REFERÊNCIAS}

1. Chiaravalloti-N eto F, Costa AIP, Soares MRD, Scandar SAS, Cardoso Junior RP. Descrição da colonização de Aedes albopictus (Diptera: Culicidae) na região de São José do Rio Preto, SP, 1991-1994. Rev Soc Bras Med Trop. 1996;29:543-8.

2. Consoli RAGB, Lourenço-de-Oliveira R. Principais mosquitos de importância sanitária no Brasil. Rio de Janeiro: Fiocruz; 1994.

3. Cunha SP, Alves JRC, Lima MM, Duarte JR, Barros LCV, da Silva JL, et al. Presença de Aedes aegypti em Bromeliaceae e depósitos com plantas no município do Rio de Janeiro, RJ. Rev Saúde Pública. 2002;36(2):244-5.

4. Forattini OP. Identificação de Aedes (Stegomyia) albopictus no Brasil. Rev Saúde Pública. 1986;20(3):244-5.

5. Forattini OP. Evolutionary epidemiological thought on infections. Rev Saúde Pública. 2002;36:257-62.

6. Gomes AC. Medidas dos níveis de infestação urbana para Aedes (Stegomyia) albopictus em programas de vigilância entomológica. Inf Epidemiol SUS. 1998;7(3):49-57.
7. Marques GRAM, Gomes AC. Comportamento antropofílico de Aedes albopictus (Skuse) (Diptera: Culicidae) na região do Vale do Paraíba, sudeste do Brasil. Rev Saúde Pública. 1997;31:125-30.

8. Marques GRAM, Forattini OP. Aedes albopictus in soil bromeliads in Ilhabela, coastal area of Southeastern Brazil. Rev Saúde Pública. 2005;39:548-552.

9. Moore CG, Mitchell CJ. Aedes albopictus in the United States: ten-year presence and public health implications. Emerg Infect Dis. 1997;3:329-34.

10. Santos RLC. Updating of the distribution of Aedes albopictus in Brazil (1997-2002). Rev Saúde Pública. 2003;37:671-3.

11. Serufo JC, O ca HM, Tavares VA, Souza AM, Rosa RV, Jamal MC, et al. Isolation of dengue virus type 1 from larvae of Aedes albopictus in Campos Altos City, state of Minas Gerais, Brazil. Mem Inst Oswaldo Cruz. 1993;88(3):503-4.

12. Sprenger $D$, Wuithiranyagool T. The discovery and distribution of Aedes albopictus in Harris Country, Texas. J Am Mosq Control Assoc. 1986;2:217-9. 